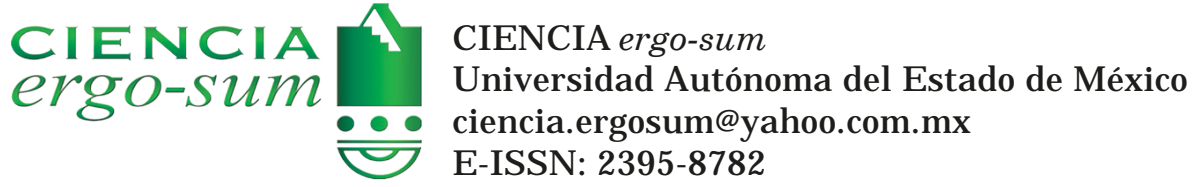

\title{
Compartir datos de investigación: reflexiones desde el ethos de la ciencia de Robert K. Merton
}

Díaz Fragoso, Omar; Riquelme Alcantar, Gabriela María Luisa; Rivera González, Gibrán

Compartir datos de investigación: reflexiones desde el ethos de la ciencia de Robert K. Merton

CIENCI A ergo-sum, vol. 29, núm. 1, marzo-junio 2022 | e147

Ciencias Sociales

Universidad Autónoma del Estado de México, México

Esta obra está bajo una Licencia Creative Commons Atribución-NoComercial-SinDerivar 4.0 Internacional .

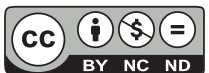

Díaz Fragoso, O., Riquelme Alcantar, G. M. L. y Rivera González, G. (2022). Compartir datos de investigación: reflexiones desde el ethos de la ciencia de Robert K. Merton. CIE NCIA ergo-sum, 29(1). https://doi.org/10.30878/ ces.v29n1a3 


\title{
Compartir datos de investigación: reflexiones desde el ethos de la ciencia de Robert K. Merton
}

\section{Sharing research data: Reflections from Robert K. Merton's ethos of science}

\author{
Omar Diaz Fragoso \\ Instituto Politécnico Nacional, México \\ omar.dz.21@gmail.com \\ (D) http://orcid.org/0000-0001-7271-9469 \\ Gabriela Maria Luisa Riquelme Alcantar \\ Instituto Politécnico Nacional, México \\ griquelme@ipn.mx \\ (1) http://orcid.org/0000-0001-7775-2938 \\ Gibrán Rivera González \\ Instituto Politécnico Nacional, México \\ gibranrg@gmail.com \\ (D) http://orcid.org/0000-0003-2805-5524
}

Recepción: 02 de junio de 2020

Aprobación: 18 de agosto de 2020

\section{RESUMEN}

Se analiza el interés de los científicos en compartir datos de investigación con base en el ethos de la ciencia de Robert K. Merton. Se revisaron treinta documentos científicos, resultado de búsquedas en bases de datos como Scopus y Web of Science; el análisis interpretativo del contenido se realizó con el programa ATLAS.ti. Entre los hallazgos destacan diversos factores asociados a la negación de los científicos para compartir datos en la ciencia. Asimismo, se encontraron otros posicionamientos que promueven una concientización a favor de distribuirlos, pues el bien común está por encima de los intereses particulares. Finalmente, se constata que el ethos es una propuesta apropiada para examinar el interés de los investigadores en la distribución de datos de investigación.

Palabras ClaVE: compartir datos, datos de investigación, ethos de la ciencia, normas de la ciencia, Robert K. Merton.

\begin{abstract}
Scientists' interest in sharing research data based on Robert K. Merton's ethos of science is analyzed. Thirty scientific documents from Scopus and Web of Science citation indexes were reviewed. The interpretative analysis of the content was done with the ATLAS. ti software. Among the findings, several factors associated with scientists' refusal to share data in science stand out. Other positions were also found that promote awareness in favor of distributing them, since the common good is above private interests. Finally, it is verified that the ethos of science is an appropriate proposal to analyze the interest of researchers in the distribution of research data.
\end{abstract}

KEYWORDS: Data sharing, research data, ethos of science, norms of science, Robert K. Merton.

\section{INTRODUCCIÓN}

En los últimos años la investigación científica ha experimentado cambios importantes. El desarrollo tecnológico, así como el uso intensivo de información incentivan un mayor acceso al quehacer científico (Borgman, 2015; Rutjens, Heine, Sutton y van Harreveld, 2018). Para lograrlo, el modelo de ciencia abierta ha sido promovido para la apertura del conocimiento científico ante cualquiera de forma transparente y reutilizable en todas las 
etapas del proceso de investigación (Bisol et al., 2014; Hampton et al., 2015). Además de impulsar el libre acceso a publicaciones, códigos, métodos y apuntes, busca que se compartan los datos de las investigaciones (Hampton et al., 2015; McKiernan et al., 2016). De hecho, naciones como Canadá (DFO, 2013), Estados Unidos (NIH, 2003; NSF, 2013) y el Reino Unido (HEFCE et al., 2016) han establecido políticas que obligan a compartir los datos generados en investigaciones financiadas con recursos públicos. Sin embargo, su adopción ha sido compleja entre los miembros de la comunidad científica (Pampel y Dallmeier-Tiessen, 2014).

En la literatura científica existen algunos estudios que exploran los factores por los que los investigadores deciden compartir datos de investigación. Los resultados permiten constatar la presencia de posicionamientos encontrados entre los científicos: algunos están a favor, mientras que otros no. Aquellos dispuestos a compartirlos consideran que los riesgos no justifican ocultarlos, dado que el beneficio resultante es para la comunidad científica y la sociedad en general. Por otro lado, quienes optan por conservarlos argumentan que los riesgos justifican no compartirlos, pues podría existir un efecto negativo para ellos. Incluso, en naciones donde compartirlos es una exigencia, se observa este fenómeno. En el caso específico de México, por ejemplo, aún no existe estímulo alguno para incentivar la participación de la academia en distribuirlos y reutilizarlos (Buenrostro, 2017; Ontiveros, 2016). Sin embargo, existe la posibilidad de que en un futuro se promueva, tal y como ya se hizo con el acceso abierto (Ley de ciencia y tecnología, 2015).

Un argumento central de este artículo es que el interés general de la sociedad debería imponerse al interés individual de los miembros de la comunidad científica. Por ello la postura en este trabajo es a favor de compartir datos de investigación. No obstante, también los argumentos de los investigadores que optan por no compartir son válidos y no deberían ser desestimados. Ante esto, se observa un debate entre lo qué es y lo qué debería ser. De ahí el cuestionamiento sobre cómo debería ser la ciencia en este aspecto: ¿Existen reglas, normas o principios sobre qué es lo correcto? Así como existen reglas técnicas para proceder en las investigaciones, ¿se cuenta con reglas que guíen el actuar de las personas encargadas de hacer ciencia?

Al respecto, una de las propuestas más conocidas es el ethos de la ciencia de Robert K. Merton (1973), la cual está asociada con la actitud que los investigadores deberían tener en torno al desarrollo y evaluación de sus hallazgos. Este autor plantea un conjunto de cuatro normas que, si bien su aplicación no es obligatoria, representan un ideal al que la ciencia debería aspirar. Sin embargo, otros aspectos también podrían ser abordados, en particular sobre el interés que tienen por compartir sus datos de investigación. Por ello, el objetivo es analizar ese interés con base en el ethos de la ciencia de Merton a fin de subrayar la relevancia que dicha propuesta podría tener en la actualidad.

\section{Marco De ReFERENCia}

En virtud de que los datos de investigación son un insumo fundamental del proceso de indagación científica, éstos se definen como registros, cuantitativos o cualitativos, de hechos que sustentan la respuesta a una pregunta de investigación y que son necesarios para validar los resultados (HEFCE et al., 2016; OECD, 2007). A manera de ejemplo, se citan "las estadísticas, mediciones, observaciones, resultados de encuestas o experimentos, registros de entrevistas e imágenes” (ERC, 2017: 6). Estos registros se convierten en datos abiertos en el momento en que "se puede acceder a ellos, usarlos, modificarlos y compartirlos libremente; siempre que exista un reconocimiento apropiado, en caso de ser necesario" (HEFCE et al., 2016: 3).

El posicionamiento de este artículo es que compartir datos de investigación es una práctica que beneficia el desarrollo de la ciencia y de sus participantes. Entre otros aspectos, contribuye a: a) acelerar el progreso científico y tecnológico (Kuipers y Van Der Hoeven, 2009; Zuiderwijk y Spiers, 2019), b) favorece la transparencia y reproducibilidad de las investigaciones científicas (Lowndes et al., 2017; Smith y Makel, 2019), c) estimula la generación de colaboraciones entre científicos (McKiernan et al., 2016; Tenopir et al., 2011) y d) ahorra recursos como dinero y tiempo de los investigadores (Poldrack y Gorgolewski, 2014; Sedransk et al., 2010). 
No obstante, en la literatura científica se constata que existen diversos factores por los que los investigadores deciden no compartir datos en la ciencia, los cuales tienen que ver con: $a$ ) la propiedad sobre los datos, $b$ ) oportunidades de publicar, $c$ ) tiempo y esfuerzo, $d$ ) reconocimiento, $e$ ) mala utilización y $f$ ) con su reputación. En este sentido, se debe considerar que para responder a las razones que obstaculizan compartir datos de investigación, así como para impulsar los argumentos que están a su favor, las instituciones científicas podrían contemplar la estructura normativa de la ciencia propuesta por Merton.

Para Merton el desarrollo científico no se da en un vacío social, pues la institución de la ciencia forma parte de una gran estructura colectiva. En 1942, este autor planteó la estructura normativa de la ciencia o ethos de la ciencia, que en su forma general está integrada por un conjunto de valores y normas que son vinculantes para el hombre de ciencia. De esta forma, este autor distingue entre dos tipos de normas: las técnicas y las éticas. Las primeras están asociadas con aspectos cognitivos y las segundas tratan sobre el comportamiento de los científicos (que es el objeto de estudio en este artículo). Cabe señalar que la transmisión de las normas éticas se da de forma implícita, es decir, que son interiorizadas por el investigador a través de un proceso de socialización con otros miembros de la comunidad científica.

Las normas éticas que integran el ethos de la ciencia comprenden el universalismo, comunismo, desinterés y escepticismo organizado (Merton, 1973). El universalismo se refiere a que la evaluación de afirmaciones científicas debe realizarse con base en criterios impersonales, en otras palabras, que no dependa de atributos personales o sociales. El comunismo indica que los investigadores deben hacer públicos sus resultados y métodos para favorecer el avance de la sociedad. El desinterés implica que entre los científicos no predomine un apego emocional o financiero respecto a sus investigaciones; su trabajo debe ser guiado por pasión al conocimiento, así como por el beneficio de la humanidad. Por último, el escepticismo organizado significa que la evaluación de los investigadores y de sus hallazgos debe ser ejecutada con base en principios empíricos y lógicos, esto es, libre de sesgos. En este aspecto, compartir los datos de investigación para transparentar el ejercicio de recursos públicos, mejorar la reproducibilidad de las investigaciones e incrementar el desarrollo de la ciencia refiere a las normas de comunismo, desinterés y escepticismo organizado.

\section{MÉTodo}

Se llevó a cabo una búsqueda de documentos en las bases de datos Web of Science y Scopus en el periodo comprendido entre 2007 y 2019, la cual arrojó 260 registros. Se utilizaron los términos data sharing, open data, research data, así como open science. El filtro contempló únicamente artículos y artículos de revisión. Con base en el objetivo, se seleccionaron los documentos relacionados con los factores que influyen en los investigadores para compartir datos de investigación. Para garantizar la pertinencia de su contenido, se revisaron el título, el resumen, así como las palabras clave y se excluyeron aquellos que no cumplieron con el criterio establecido; asimismo, se descartaron los que, hasta ese momento, no habían recibido cita alguna debido a que aún no es clara su relevancia en el tema.

La muestra final se refinó a treinta documentos que se abordaron a profundidad por medio de un análisis interpretativo, con el apoyo informático de ATLAS.ti; la intención era identificar los factores que, desde la perspectiva de los investigadores y sin distinción de disciplina, inhiben compartir datos en la ciencia. Destacan los individuales que dependen de los intereses y riesgos percibidos por los propios investigadores. Por otro lado, se encuentran los técnicos e institucionales que no fueron considerados debido a que los científicos no tienen control ni poder de decisión sobre ellos. De igual forma, se ubicaron los argumentos que los promotores de la ciencia abierta utilizan para fomentar una mayor apertura del quehacer científico, particularmente, los asociados con compartir datos de investigación. La codificación fue de tipo abierta (Braun y Clarke, 2006), es decir, que las categorías de análisis emergieron a partir de la revisión de los artículos científicos elegidos y procesados. Por último, los resultados obtenidos se interpretaron con base en el contenido de la propuesta de Merton (1973) sobre la estructura normativa de la ciencia a fin de mostrar la importancia de que las instituciones consideren retomar y promover las normas éticas planteadas por este autor en aras de lograr distribuir datos en la ciencia. 


\section{Resultados Y Discusión}

En primera instancia, en este apartado se abordan los factores por los que los investigadores prefieren no compartir datos de investigación con la comunidad científica y el resto de la sociedad. Después, se exhiben las razones por las que se deberían compartir los datos científicos de acuerdo con los promotores de la ciencia abierta. Por último, se propone que para estimular la distribución de datos de investigación podría ser de utilidad tomar como referente la estructura normativa de la ciencia propuesta por Merton.

\section{1. Factores que inhiben compartir datos de investigación}

El análisis interpretativo del contenido de los artículos elegidos permitió identificar siete factores de tipo individual que los investigadores perciben que atentan contra sus intereses y que, por ende, desincentivan a compartir datos de investigación: a) Considerar los datos como propiedad privada, $b$ ) perder oportunidades de publicar, c) tiempo y esfuerzo requeridos para compartir, $d$ ) falta de reconocimiento por compartir, $e$ ) mal uso que otros puedan hacer de los datos, $f$ ) revelar errores cometidos por los investigadores y $g$ ) las consecuencias en la reputación de quien lo hace al distribuirlos. Enseguida, se presenta cada uno de ellos.

Algunos investigadores consideran que los datos generados en sus proyectos son propiedad privada y que por ello no tienen obligación de compartirlos con la comunidad científica o la sociedad. En efecto, Nguyen et al. (2017) sostienen que algunos científicos afirman ser propietarios de los datos, puesto que son quienes se han esforzado y absorbido los costos de su recolección. Justamente, Zuiderwijk y Spiers (2019) lo reafirman al mencionar que en disciplinas donde el investigador efectúa el trabajo de campo para colectar los datos tiende a existir mayor sentido de pertenencia sobre los mismos. En adición, la propiedad en torno a éstos se complica aún más cuando los investigadores desconocen la legislación acerca de derechos de autor en sus países de origen (Fecher et al., 2015), como es el caso de las colaboraciones entre investigadores (Cragin et al., 2010; Zuiderwijk \& Spiers, 2019).

Otros científicos argumentan que estarían dispuestos a compartir sus datos de investigación siempre y cuando tuvieran el control de la información generada o al menos conocimiento de sus futuras utilizaciones (Pitt y Tang, 2013; Wallis, Rolando y Borgman, 2013). En resumen, el sentido de pertenencia sobre los datos está estrechamente asociado con la forma como fueron colectados y el control que los investigadores desean tener sobre éstos últimos.

Otra de sus preocupaciones pone el acento en la difusión de sus hallazgos de manera anticipada por parte de aquellos con quienes compartieron sus datos de investigación con la consabida pérdida de oportunidades de publicación. En un estudio hecho por Andreoli-Versbach y Mueller-Lager (2014), en donde revisan la actitud de investigadores en economía y administración en torno al tema, se identificó que compartir datos es una forma de competencia autoinducida, ya los investigadores poseedores del conocimiento permiten que otros publiquen hallazgos antes de que sus creadores los exploten completamente.

En este orden de ideas, Bisol et al. (2014) argumentan que en el Programa del Genoma Humano (HGP por sus siglas en inglés) el principal factor que inhibe que los científicos compartan sus datos es el temor de no tener oportunidad de analizarlos en su totalidad y de preparar sus publicaciones. Finalmente, Wallis, Rolando y Borgman (2013) describen que investigadores del Centro de Detección de Redes Integradas (CENS por sus siglas en inglés) estarían dispuestos si sus creadores retuvieran el derecho de ser los primeros en hacerlo; de lo contrario, se sentirían despojados si otros publicaran hallazgos que hicieran irrelevantes a los suyos.

Luego, la cantidad de tiempo, así como el esfuerzo requerido son otras razones que esgrimen algunos investigadores renuentes a curar y compartir sus datos de investigación. Zuiderwijk y Spiers (2019) muestran que para algunos científicos en astrofísica el tiempo y esfuerzo necesarios para prepararlos y compartirlos es desalentador, porque a menudo tienen otras tareas por hacer en el ámbito investigativo. Esto se complica aún más en disciplinas pertenecientes a las ciencias sociales y las humanidades por la ausencia de estándares en 
torno a los metadatos, así como a la falta de procedimientos establecidos sobre compartir datos científicos, lo cual implica que sea costoso y requiera demasiado tiempo (Nguyen et al., 2017). Incluso, algunos científicos argumentan que contar con el apoyo de alguien para curar sus datos facilitaría su distribución. De igual forma, Cragin et al., (2010) sostienen que para algunos investigadores recibir varias solicitudes para que compartan sus datos se convierte en una carga insostenible, ya que además cumplen con otras obligaciones como impartir clases o asistir a eventos académicos y de investigación. Referente a esto, no sólo les preocupa el tiempo y esfuerzo requeridos para compartirlos sino que también les incomoda el esfuerzo adicional que se necesita para apoyar a quienes reutilizan sus datos (Fecher et al., 2015). En suma, la naturaleza de los datos colectados en cada área del conocimiento, al igual que la falta de personal de apoyo, incide en el tiempo y esfuerzo requerido para su preparación y distribución.

De hecho, los argumentos expuestos se arraigan más entre los investigadores al momento de no ser reconocidos por compartir sus datos de investigación. Esta falta de reconocimiento se refiere a que, para los científicos, en el sistema académico actual no existen incentivos o recompensas por generar, curar y compartir datos de investigación (Bisol et al., 2014). En un estudio que llevaron a cabo Sayogo y Pardo (2013), donde se explora el interés de algunos investigadores por compartirlos, se constata que uno de los factores determinantes que inhibe su distribución es la ausencia de crédito y reconocimiento para quien los comparte. Por tanto, los investigadores prefieren mantener control absoluto sobre los datos que generan. Ciertamente, esta situación se acentúa entre investigadores jóvenes debido a que el sistema académico no reconoce prácticas asociadas a compartir datos; en cambio, da prioridad a publicar artículos, que es lo que permite su avance en la jerarquía académica (Pfenninger et al., 2017). Del mismo modo, Choudhury et al., (2014) describen que en la comunidad neurocientífica no se incentiva esta actividad, pues predomina una cultura competitiva en donde se reconoce ser el primero en analizarlos y publicar hallazgos a partir de éstos.

Otro aspecto importante que desincentiva a los investigadores está relacionado con el mal uso que otros investigadores pueden hacer de ellos. Fecher et al., (2015) identifican que el mal uso de los datos se puede dar de distintas formas como falsificación, uso comercial, mal uso competitivo e interpretación defectuosa. En el caso de las humanidades, Kuipers y Van Der Hoeven (2009) refieren que quienes comparten datos temen que futuros usuarios no sean capaces de entenderlos y, por ende, se haga una mala interpretación. Sobre todo, Cooper (2007) señala que compartir datos sensibles puede dañar la integridad de los sujetos estudiados. Además, algunos científicos prefieren reservarlos porque les inquieta que otros individuos o empresas privadas los exploten para obtener un beneficio comercial (Costello, 2009). Peor aún, Pitt y Tang (2013) sostienen que un mal uso competitivo de datos se da cuando algunos individuos los reutilizan para buscar discrepancias en el trabajo de los científicos que los comparten.

Ahora bien, otro de los factores que inhibe que los investigadores compartan sus datos es la puesta en evidencia de errores en el proceso de investigación. En efecto, Zuiderwijk y Spiers (2019) hacen notar que en astrofísica a algunos científicos les desagrada que sus investigaciones sean sometidas a un escrutinio detallado. Principalmente, algunos prefieren ocultar sus datos cuando sus publicaciones contienen análisis estadísticos que son propensos a error (Bisol et al., 2014), es decir, les preocupa que se exponga la baja calidad de su método de recolección o de análisis (Choudhury et al., 2014; Sayogo y Pardo, 2013). De hecho, Andreoli-Versbach y Mueller-Langer (2014) sugieren que el temor asociado con la revelación de errores también está asociado con la jerarquía o posición del investigador. Referente a esto, los científicos jóvenes suelen cometer más errores que los experimentados y, por consiguiente, prefieren ser más cautelosos al compartir sus datos de investigación.

Asimismo, el mal uso que otros pueden hacer de los datos compartidos, así como la búsqueda de errores en el quehacer científico, tienen un efecto negativo en la reputación del investigador, que sucede cuando se comparten datos inutilizables por estar incompletos o ser de baja calidad y por lo cual la reputación se verá afectada (Zuiderwijk y Spiers, 2019). De igual forma, Cragin et al., (2010) indican que la interpretación incorrecta por parte de otros científicos tiene un efecto negativo sobre el renombre del investigador que los colectó. Peor aún, 
la fama de los científicos que comparten sus datos se podría ver afectada cuando quien los reutiliza encuentra otros resultados o aspectos que el investigador primario omitió en su análisis (Sayogo y Pardo, 2013). Como se observa, la distribución de sus datos representa para algunos investigadores la pérdida de control y la posibilidad de ser sujetos a la crítica y humillación por parte de otros (Choudhury et al., 2014; Pitt y Tang, 2013).

\section{2. Argumentos para promover la distribución de datos científicos}

No obstante que en la literatura científica existen diversos ejemplos que dan cuenta de los beneficios asociados con la apertura del quehacer científico (Childs et al., 2014; Lowndes et al., 2017; McKiernan et al., 2016; Zuiderwijk y Spiers, 2019), se puede constatar que son diversos, así como legítimos, los riesgos que los investigadores perciben respecto a compartir datos de investigación en la ciencia. En consecuencia, es díficil lograr que en la práctica los científicos lleven a cabo voluntariamente la distribución. Sin embargo, en este artículo se exploran tres argumentos citados a menudo por los promotores de la ciencia abierta y de los datos abiertos para defender su causa. El primero tiene que ver con el financiamiento público que reciben las investigaciones; el segundo, con una mayor transparencia y escrutinio del quehacer científico y, el último, con el avance del conocimiento científico. A continuación, se aborda cada uno de ellos.

En primer lugar, al igual que otros sectores de la sociedad, la academia no debería ser excluida de la aplicación de algunos mecanismos de transparencia en torno al ejercicio de fondos públicos. En este sentido, los argumentos vertidos en el artículo coinciden con Borgman (2012) en lo que corresponde a que los datos producidos con fondos públicos tendrían que ser abiertos y no ser acaparados por los investigadores, pues lo lógico es que el dinero de los impuestos sea aprovechado para servir al bien común. En efecto, Janssen et al., (2012) reflexionan acerca de que compartir datos de investigación permitiría la generación de riqueza a través del uso de sus productos, proporcionaría a los políticos datos para abordar problemas complejos, involucraría a la ciudadanía en el análisis y a su vez, más importante aún, promovería la ciencia ciudadana (Borgman, 2012). Pero, sobre todo, representaría la oportunidad de que los contribuyentes observaran en donde se están invirtiendo los impuestos recaudados (Nguyen et al., 2017). Por esta razón, existen distintos pronunciamientos y políticas que exigen la apertura de la actividad científica, particularmente de los datos en la ciencia (Brown et al., 2003; Bullinger et al., 2003; Chan et al., 2002; ERC, 2017; HEFCE et al., 2016; Murray-Rust et al., 2010; NIH, 2003; NSF, 2013; OECD, 2007).

Recientemente, en la ciencia se han suscitado algunos problemas de fraude y de reproducibilidad en relación con algunos hallazgos científicos (Kim y Kim, 2018; Vinck, 2014; Wicherts, 2011). De este modo, tanto la comunidad científica como la sociedad demandan una mayor transparencia y escrutinio del quehacer investigativo, por lo que compartir datos de investigación forma parte de la solución. Para Poldrack y Gorgolewski (2014) compartirlos permite mejorar la confiabilidad y reproducibilidad de las investigaciones al hacer posible analizar y verificar, de forma independiente, los hallazgos de trabajos publicados previamente (Nguyen et al., 2017; Tenopir et al., 2011; Wiley y Mischo, 2016). Por consiguiente, se estará en condiciones de refutar o confirmar los resultados de las investigaciones (Link et al., 2017). Incluso, el escrutinio del proceso de investigación incentiva a los científicos a no caer en malas prácticas y mejorar la calidad de sus trabajos (Nguyen et al., 2017; Pfenninger et al., 2017; Zuiderwijk y Spiers, 2019).

Aunado a lo anterior, la transparencia del quehacer científico es complementada por una aceleración en el progreso de la ciencia. En este sentido, existen diversos testimonios respecto a compartir datos de investigación y el efecto que tiene en el avance científico. De hecho, re-utilizar datos de investigación permite que los investigadores construyan nuevos hallazgos sobre la base de conocimiento ya existente (Harper y Kim, 2018; Kuipers y Van Der Hoeven, 2009; Wallis, Rolando y Borgman, 2013). Asimismo, brinda la oportunidad de abordar los datos con nuevas preguntas, métodos y técnicas de análisis (Nguyen et al., 2017; Pitt y Tang, 2013; Poldrack y Gorgolewski, 2014) prolongando su vida útil (Link et al., 2017). Luego, es posible integrar conjuntos de datos con el propósito de buscar solución a preguntas y/o problemáticas más complejas (Bisol et al., 2014; Choudhury 
et al., 2014; Nguyen et al., 2017). Por último, es probable que los científicos mejoren sus investigaciones al recibir retroalimentación sobre la forma en que han colectado y procesado los datos de sus investigaciones (Cragin et al., 2010; Zuiderwijk y Spiers, 2019).

En general, se puede constatar que los motivos por los que los investigadores estarían obligados a compartir sus datos de investigación apelan a una concientización de que el bien común debería prevalecer sobre los intereses particulares. Si bien es cierto que han surgido distintos pronunciamientos, campañas y políticas para promover el libre acceso a la ciencia, su implementación y adopción ha sido lenta debido a la dinámica que está presente en el sistema académico; sin embargo, es necesario que sus miembros empiecen a entender que la ciencia no es una entidad ajena a la sociedad (Olivé, 2007).

\section{3. Compartir datos de investigación desde el ethos de la ciencia}

En este artículo se sostiene que para promover la distribución de datos de investigación, las instituciones científicas podrían considerar las normas éticas propuestas por Merton. En relación con esto, compartir los datos de investigación para transparentar el ejercicio de recursos públicos, mejorar la reproducibilidad de las investigaciones e incrementar el desarrollo de la ciencia constituye prestar atención a las normas de comunismo, desinterés y escepticismo organizado.

También de especial interés es el transparentar el uso de recursos pertenecientes a las investigaciones científicas, que se encuentra asociado con la norma llamada comunismo. Y es que, en el caso de la ciencia, transparentar no se podría limitar a los recursos financieros ejercidos en las investigaciones, puesto que desde la perspectiva mertoniana contempla los insumos y sus productos. Por este motivo, los datos de investigación podrían ser considerados como un bien público al que otros pueden acceder y hacer uso. Dicho de otra manera, no debería buscarse su privatización y, menos aún, su comercialización. De lo contrario, se pervierte su manejo, ya que el conocimiento pasa a ser tratado como una mercancía. Además, poner en práctica el comunismo permite que el potencial de los datos de investigación vaya más allá de las comunidades científicas. En este tenor, sirven también de utilidad para empresarios, gobernantes y ciudadanos quienes pueden emplearlos para dar solución a alguna problemática de su comunidad. A nuestro juicio, tratar los datos de investigación como un bien común aumentaría su posible utilidad.

Asimismo, tanto el fomento del escrutinio como la mejora de la reproducibilidad de las investigaciones están asociados con el escepticismo organizado. Tener acceso a los datos de investigación favorece la revisión detallada de los insumos empleados para obtener resultados. El hecho de poseer los datos permite que los cálculos, experimentos y simulaciones sean replicados y así garantizar la certeza de las investigaciones. En caso de no ser así, los datos permitirían su refutación, la cual se realizaría con base en criterios lógicos y empíricos tal y como lo propuso Merton. Adicionalmente, probar que los resultados de una investigación son verdaderos refuerza la legitimidad de los científicos y de sus propuestas. Por eso, en este artículo se argumenta sobre la importancia de que los investigadores sometan sus trabajos a escrutinio, pues garantiza que su reputación se construya a partir de la calidad de sus aportaciones y no por otra clase de criterios.

De esta forma, promover el desarrollo de la ciencia representa la concordancia con la norma denominada desinterés. De acuerdo con Merton (1973), el objetivo de la ciencia es el avance del conocimiento científico. Por ello, el hecho de que el investigador comparta datos de investigación para que otros los aprovechen constituye un acto admirable al dejar de lado ciertos intereses que podrían resultar en un beneficio personal e inmediato tales como publicar artículos, presentarse en conferencias o recibir citas. Por el contrario, el científico brinda la posibilidad de que otros usuarios que poseen una mirada de investigación diferente puedan hacer uso de dichos datos y llegar a resultados que probablemente no hubiera alcanzado o a que contribuyan a resolver problemas pendientes. Lo anterior constituye un cambio de visión, pues los miembros de la comunidad científica dejan de ver la ciencia como un esfuerzo individual para observarla como un esfuerzo colectivo cuyo objetivo es el progreso del conocimiento en beneficio de la sociedad en general. 


\section{AnÁlisis PRospectivo}

No obstante que el ethos de la ciencia es una idealización (Bray y von Storch, 2017; Kim y Kim, 2018) y la promoción de la ciencia abierta es reciente, se puede constatar que el primero es afín a los argumentos por los que los investigadores estarían interesados en compartir sus datos de investigación. Más aún, suponiendo que los científicos antepusieran el beneficio común al propio, funcionan como respuesta ante las razones por las que prefieren preservar sus datos. Sin embargo, el escenario futuro se vislumbra complicado, ya que en el sistema académico actual predominan prácticas que representan lo contrario.

Desde hace tiempo, la ciencia se rige por el modelo capitalista que prioriza la generación de riqueza por encima del avance del conocimiento (Krishna, 2014; Slaughter y Rhoades, 2004). De igual forma, los compromisos comerciales entre universidades y/o centros de investigación con el gobierno e industria obligan a los investigadores a ocultar los productos de sus investigaciones (Bray y von Storch, 2017). En efecto, son ocultados con el objetivo de patentarlos y comercializarlos en el mercado (Slaughter y Rhoades, 2004). Por lo tanto, la investigación se encuentra atrapada por intereses comerciales, dado que los investigadores alinean sus proyectos de acuerdo con los de los financiadores (Macfarlane y Cheng, 2008). Lo que es peor: los científicos se ven forzados a ocultar sus datos porque la presión por ascender en la escalera social los obliga a publicar sus hallazgos en revistas científicas de alto impacto; de lo contrario, son invisibilizados (Publish or Perish) (Vinck, 2014).

Desde nuestra perspectiva, para cambiar el estado actual del sistema académico, es necesario que las universidades, centros de investigación, financiadores y sociedades científicas establezcan medidas que incentiven a los investigadores a compartir sus datos y a entender los beneficios que su distribución implica. Ciertamente, además de beneficiar a otros, los obliga a desarrollar investigaciones de mayor calidad al saber que sus trabajos serán sometidos a un mayor escrutinio científico. En fin, se requiere de un cambio de perspectiva donde el manejo de datos pase de ser entendido como un medio para producir publicaciones a un fin en sí mismo que puede tener mayor impacto en la comunidad científica y en la sociedad en general.

\section{Conclusiones}

En conclusión, compartir datos de investigación es un esfuerzo loable que favorece la transparencia y avance del quehacer científico. Las razones que sus promotores proporcionan son valiosas, ya que anteponen el beneficio común. Sin embargo, también resultan legítimas las inquietudes de los investigadores en torno a compartir datos; más aún, cuando el sistema académico estimula la ejecución de prácticas que implican lo opuesto a la apertura del proceso de investigación. Entonces, se constata que en la ciencia predominan los secretos, los particularismos, así como los intereses que se oponen al ethos de la ciencia. No obstante, este tipo de debates sobre la relación ciencia-sociedad son importantes, pues retoman propuestas como la estructura normativa de la ciencia planteada por Merton que obligan a cuestionarse acerca del estado actual de la institución científica. Se esperaría que los investigadores reflexionen y consideren los beneficios que una ciencia abierta puede tener para la sociedad y también en un nivel individual. Asimismo, será importante que los gobiernos, consejos de investigación, centros de investigación, así como universidades establezcan políticas y mecanismos de evaluación que estimulen compartir datos dentro de sus comunidades científicas.

\section{Agradecimientos}

Se agradece a la Secretaría de Investigación y Posgrado del Instituto Politécnico Nacional que auspició los proyectos de investigación 20211020, 20221371, 20211084 y 20220403 para realizar este artículo, al Fondo Sectorial de Investigación para la Educación del Conacyt por el apoyo al proyecto A1-S-46300 y se agradece a los evaluadores del artículo pues sus observaciones permitieron mejorar, así como enriquecer su estructura. 


\section{REFERENCIAS}

Andreoli-Versbach, P., \& Mueller-Langer, F. (2014). Open access to data: An ideal professed but not practised. Research Policy, 43(9), 1621-1633. https://doi.org/10.1016/j.respol.2014.04.008

Bisol, G. D., Anagnostou, P., Capocasa, M., Bencivelli, S., Cerroni, A., Contreras, J., \& ... Boulton, G. (2014). Perspectives on open science and scientific data sharing: An interdisciplinary workshop. Journal of Anthropological Sciences, 92(2014), 179-200. https://doi.org/10.4436/JASS.92006

Borgman, C. L. (2012). The conundrum of sharing research data. Journal of the American Society for Information Science and Technology, 63(6), 1059-1078. https://doi.org/https://doi.org/10.1002/asi.22634

Borgman, C. L. (2015). Big data, little data, no data: Scholarship in the networked world. Cambridge: MIT press.

Braun, V., \& Clarke, V. (2006). Using thematic analysis in psychology. Qualitative Research in Psychology, 3(2), 77-101. https://doi.org/https://doi.org/10.1191/1478088706qp063oa

Bray, D., \& von Storch, H. (2017). The normative orientations of climate scientists. Science and Engineering Ethics, 23(5), 13511367. https://doi.org/10.1007/s11948-014-9605-1

Brown, P. O., Cabell, D., Chakravarti, A., Cohen, B., Delamothe, T., Eisen, M., \& ... Johnson, R. K. (2003). Bethesda statement on open access publishing.

Buenrostro, E. (2017). La generación de valor a partir de los datos abiertos de gobierno. Disponible en https:// www.infotec.mx/es_mx/infotec/la_generacion_de_valor_a_partir_de_los_datos_abiertos_de_gobierno

Bullinger, H.-J., Einhäupl, K. M., Gaehtgens, P., Gruss, P., Henkel, H.-O., Kröll, W., ... Galluzzi, P. (2003). Berlin declaration on open access to knowledge in the sciences and humanities. Open Access to Knowledge in the Sciences and Humanities, 20-22.

Chan, L., Cuplinkas, D., Eisen, M., Friend, F., Genova, Y., Guédon, J.-C., \& ... Velterop, J. (2002). Budapest Open Access Initiative. Disponible en https://www.budapestopenaccessinitiative.org/read

Childs, S., McLeod, J., Lomas, E., \& Cook, G. (2014). Opening research data: Issues and opportunities. Records Management Journal, 24(2), 142-162. https://doi.org/10.1108/RMJ-01-2014-0005

Choudhury, S., Fishman, J. R., McGowan, M. L., \& Juengst, E. T. (2014). Big data, open science and the brain: Lessons learned from genomics. Frontiers in Human Neuroscience, 8, 1-10. https://doi.org/10.3389/fnhum.2014.00239

Cooper, M. (2007). Sharing data and results in ethnographic research: Why this should not be an ethical imperative. Journal of Empirical Research on Human Research Ethics, 2(1), 3-19. https://doi.org/https://doi. org/10.1525/jer.2007.2.1.3

Costello, M. J. (2009). Motivating online publication of data. BioScience, 59(5), 418-427. https://doi. org/10.1525/bio.2009.59.5.9

Cragin, M. H., Palmer, C. L., Carlson, J. R., \& Witt, M. (2010). Data sharing, small science and institutional repositories. Philosophical Transactions of the Royal Society A: Mathematical, Physical and Engineering Sciences, 368(1926), 4023-4038. https://doi.org/10.1098/rsta.2010.0165

DFO. (2013). Policy for Scientific Data. Retrieved from http://www.dfo-mpo.gc.ca/science/publications/datapolicy-politiquedonnees/index-eng.html\#7-1

ERC. (2017). Guidelines on implementation of open access to scientific publications and research data in projects supported by the european research council under horizon 2020. Retrieved from https://ec.europa.eu/research/ participants/data/ref/h2020/other/hi/oa-pilot/h2020-hi-erc-oa-guide_en.pdf

Fecher, B., Friesike, S., \& Hebing, M. (2015). What drives academic data sharing? PLOS ONE, 10(2), e0118053. https://doi.org/10.1371/journal.pone.0118053 
Hampton, S. E., Anderson, S. S., Bagby, S. C., Gries, C., Han, X., Hart, E. M., \& ... Zimmerman, N. (2015). The Tao of open science for ecology. Ecosphere, 6(7), https://doi.org/10.1890/es14-00402.1

Harper, L. M., \& Kim, Y. (2018). Attitudinal, normative, and resource factors affecting psychologists' intentions to adopt an open data badge: An empirical analysis. International Journal of Information Management, 41(2018), 23-32. https://doi.org/10.1016/j.ijinfomgt.2018.03.001

HEFCE, RCUK, Universities UK (UUK), \& Wellcome. (2016). Concordat On Open Research Data. Retrieved from http://www.rcuk.ac.uk/documents/documents/concordatonopenresearchdata-pdf/

Janssen, M., Charalabidis, Y., \& Zuiderwijk, A. (2012). Benefits, adoption barriers and myths of open data and open government. Information Systems Management, 29(4), 258-268. https://doi.org/10.1080/1 0580530.2012 .716740

Kim, S. Y., \& Kim, Y. (2018). The ethos of science and its correlates: An empirical analysis of scientists' endorsement of Mertonian norms. Science, Technology and Society, 23(1), 1-24. https://doi. org/10.1177/0971721817744438

Krishna, V.V. (2014). Changing social relations between science and society: Contemporary challenges. Science, Technology and Society, 19(2), 133-159. https://doi.org/10.1177/0971721814529876

Kuipers, T., \& Van Der Hoeven, J. (2009). PARSE . Insight Insight into digital preservation of research output in Europe. Retrieved from http://www.parse-insight.eu/downloads/PARSE-Insight_D3-4_SurveyReport_final_hq.pdf

Ley de ciencia y tecnología (2015). Ciudad de México: Cámara de Diputados del H. Congreso de la Unión.

Link, G. J., Lumbard, K., Conboy, K., Feldman, M., \& Feller, J. (2017). Contemporary issues of open data in information systems research: Considerations and recommendations. Communications of the Association for Information Systems, 41, 587-610. https://doi.org/https://doi.org/10.17705/1CAIS.04125

Lowndes, J. S. S., Best, B. D., Scarborough, C., Afflerbach, J. C., Frazier, M. R., O’Hara, C. C., \&... Halpern, B. S. (2017). Our path to better science in less time using open data science tools. Nature Ecology and Evolution, 1(6). https://doi.org/10.1038/s41559-017-0160

Macfarlane, B., \& Cheng, M. (2008). Communism, universalism and disinterestedness: Re-examining contemporary support among academics for Merton's scientific norms. Journal of Academic Ethics, 6(1), 67-78. https://doi.org/10.1007/s10805-008-9055-y

McKiernan, E. C., Bourne, P. E., Brown, C. T., Buck, S., Kenall, A., Lin, J., \& ... Yarkoni, T. (2016). How open science helps researchers succeed. eLife, 5, 1-19. https://doi.org/10.7554/eLife.16800

Merton, R. K. (1973). The sociology of science: Theoretical and empirical investigations. Chicago: University of Chicago Press.

Murray-Rust, P., Neylon, C., Pollock, R., \& Wilbanks, J. (2010). Panton principles, principles for open data in science. Panton Principles.

Nguyen, V. M., Brooks, J. L., Young, N., Lennox, R. J., Haddaway, N., Whoriskey, F. G., \&... Cooke, S. J. (2017). To share or not to share in the emerging era of big data: Perspectives from fish telemetry researchers on data sharing. Canadian Journal of Fisheries and Aquatic Sciences, 74(8), 1260-1274. https://doi.org/https:// doi.org/10.1139/cjfas-2016-0261

NIH. (2003). NIH data sharing policy and implementation guidance. Retrieved fromhttps://grants.nih.gov/ grants/policy/data_sharing/data_sharing_guidance.htm

NSF. (2013). Open Data at NSF. Retrieved from https://www.nsf.gov/data/

OECD. (2007). OECD Principles and guidelines for access to research data from public funding. 
Olivé, L. (2007). La ciencia y la tecnología en la sociedad del conocimiento. Ciudad de México: Fondo de Cultura Económica.

Ontiveros, M. (2016). Datos abiertos de investigación. Caso México. Ciudad de México: Conacyt.

Pampel, H., \& Dallmeier-Tiessen, S. (2014). Open research data: From vision to practice. In S. Friesike \& S. Bartling (eds.), Opening science the evolving guide on how the internet is changing research, collaboration and scholarly publishing (pp. 213-224). Cham: Springer Open.

Pfenninger, S., DeCarolis, J., Hirth, L., Quoilin, S., \& Staffell, I. (2017). The importance of open data and software: Is energy research lagging behind? Energy Policy, 101, 211-215. https://doi.org/10.1016/j. enpol.2016.11.046

Pitt, M. A., \& Tang, Y. (2013). What should be the data sharing policy of cognitive science? Topics in Cognitive Science, 5(1), 214-221. https://doi.org/10.1111/tops.12006

Poldrack, R. A., \& Gorgolewski, K. J. (2014). Making big data open: Data sharing in neuroimaging. Nature Neuroscience, 17(11), 1510-1517. https://doi.org/10.1038/nn.3818

Rutjens, B. T., Heine, S. J., Sutton, R. M., \& van Harreveld, F. (2018). Attitudes towards science. Advances in Experimental Social Psychology, 57, 125-165.https://doi.org/https://doi.org/10.1016/bs.aesp.2017.08.001

Sayogo, D. S., \& Pardo, T. A. (2013). Exploring the determinants of scientific data sharing: Understanding the motivation to publish research data. Government Information Quarterly, 30, S19-S31. https://doi. org/10.1016/j.giq.2012.06.011

Sedransk, N., Young, L. J., Kelner, K. L., Moffitt, R. A., Thakar, A., Raddick, J., \& ... Spiegelman, C. (2010). Make research data public?-Not Always so Simple: A dialogue for statisticians and science editors. Statistical Science, 25(1), 41-50. https://doi.org/10.1214/10-sts320

Slaughter, S., \& Rhoades, G. (2004). Academic capitalism and the new economy: Markets, state, and higher education. Baltimore: The Johns Hopkins University Press.

Smith, K. N., \& Makel, M. C. (2019). Open science: A candid conversation. Journal of Advanced Academics, 30(2), 111-123. https://doi.org/https://doi.org/10.1177/1932202X19829750

Tenopir, C., Allard, S., Douglass, K., Aydinoglu, A. U., Wu, L., Read, E., \&... Frame, M. (2011). Data sharing by scientists: Practices and perceptions. PLOS ONE, 6(6), e21101. https://doi.org/10.1371/journal. pone.0021101

Vinck, D. (2014). Ciencias y sociedad. Sociología del trabajo cientifico. Barcelona: Editorial Gedisa.

Wallis, J. C., Rolando, E., \& Borgman, C. L. (2013). If we share data, will anyone use them? Data sharing and reuse in the long tail of science and technology. PLOS ONE, 8(7), e67332. https://doi.org/https://doi. org/10.1371/journal.pone.0067332

Wicherts, J. M. (2011). Psychology must learn a lesson from fraud case. Nature, 480(7375), 7. https://doi. org/10.1038/480007a

Wiley, C., \& Mischo, W.H. (2016). Data management practices and perspectives of atmospheric scientists and engineering faculty. Issues in Science and Technology Librarianship, 85, 1-7.https://doi.org/10.5062/F43X84NJ

Zuiderwijk, A., \& Spiers, H. (2019). Sharing and re-using open data: A case study of motivations in astrophysics. International Journal of Information Management, 49, 228-241. https://doi.org/10.1016/j.ijinfomgt.2019.05.024

\section{BY-NC-ND}

